"Placebo." If one compares the mean pretreatment blood pressure with mean "placebo" blood pressure in Table 1 there is a fall in blood pressure of $26 / 9 \mathrm{~mm}$. $\mathrm{Hg}$ which is very similar to our own results, $26 / 10 \mathrm{~mm}$. $\mathrm{Hg}$, when patients were receiving a diuretic alone.

The authors consider various explanations for the failure of the blood pressure to rise to untreated levels when propranolol was stopped. As almost all were still on diuretic therapy during this time it would seem that no other explanation need be considered.

The results presented in Fig. 1 of their paper suggest that the maximal effect of propranolol is seen in less than six weeks, and does not support their initial hypothesis that some trials have failed to show an effect of propranolol because the duration of treatment was not long enough.

The authors say that they selected the 32 patients for study from more than 300 on the drug, the grounds of selection being that they had responded to propranolol in a dose of less than $320 \mathrm{mg}$. daily. If patients are selected for inclusion in a trial on the ground that they had already responded well to the drug the result is bound to be biased in favour of the drug under study, and no amount of care in trial design will eliminate this bias.-We are, etc.

$$
\text { C. T. DOLleRY. }
$$

Department of Clinical Pharmacology, London $\mathrm{W} .12$.

J. W. Paterson.

Institute of Diseases of the Chest,

London S.W.3.

REFERENCE

1 Paterson, J. W. and Dollery, C. T. Lancet, 1966, 2,1148 .

\section{Tetrabenazine for Extra-pyramidal Movement Disorders}

SIR,-Dr. W. A. G. MacCallum (21 March, p. 760) expresses the hope that tetrabenazine may well be an alternative in some cases to L-dopa. I do not feel that the pharmacological effects of tetrabenazine warrant such a statement. Though tetrabenazine reduces choreiform movements, this effect does not minimize the possibility of production of other extra-pyramidal symptoms. An analogy exists in that drugs such as reserpine, phenothiazines, and butyrophenones, which reduce the choreiform movements of, for instance, Huntington's chorea, are themselves capable of producing severe extra-pyramidal sideeffects.

Dalby ${ }^{1}$ noted that in contrast to the beneficial effect of tetrabenazine in choreiform and hemiballistic motor activity, patients with Parkinsonian tremors and those with intention tremors due to spinocerebellar degeneration experienced the opposite effect. Thus, the use of the drug is limited, and as other drugs have an equivalent amount of effect in controlling choreiform movements, there is no benefit in resurrecting the drug. Tetrabenazine, being a benzoquinolizine derivative, resembles reserpine in chemical structure and pharmacological and biochemical actions leading to depletion of noradrenaline and serotonin concentrations in the brain, and its side effects such as depression, postural hypotension, and extra-pyramidal symptoms are similar to those of reserpine.
Owing to these side effects caution must be exercised before re-introducing it into clinical practice.-I am, etc.,

Saxondale Hospital,

R. Thavasothy.

Nottingham.

\section{REFERENCE}

42 , M. A., British Medical fournal, 1969, 2,

\section{Long-term Anticoagulant Treatment after Myocardial Infarction}

SIR,-Your leading article entitled "Long-term Anticoagulant Treatment after Myocardial Infarction (28 February, p. 514) seems to me so one-sided that it is difficult to believe it was written by an "unbiased observer".

The author referred to the work of the international review group (an analysis of the results on nine controlled trials between 1950 and 1965) in one sentence "... [they] could not reach an agreed decision." This in a way is correct, but certainly leaves the reader with an incorrect impression of what the international group said. Permit me to repeat that the group found a $20 \%$ reduction in mortality in men in the anticoagulant treated group compared to the control group. The mortality per 100 patients per month was 0.63 against 0.79 , respectively. This difference is statistically significant $(p<0.01)$. The effect seemed to last several years in both older and younger men. The group concluded "Most of us considered that the consistency of the findings warranted a conclusion that anticoagulant therapy probably prolonged survival at least over two years . . .". "Two of us were not convinced that long-term therapy prolonged survival." It is worth mentioning that the two were authors of one of the nine trials under review.

Your leader writer states: "G. Aspenstrøm and $K$. Korsen-Bengsten concluded that there was no difference in mortality between patients treated with long-term anticoagulants and untreated controls." What they actually found was 39 deaths in the treated group against 50 deaths in the control group, and their own conclusion was that ". . . the mortality through second to fifth years was in all probability significantly reduced in poor risk patients on dicumarol $(p<0.05)$.",

You state: "E. A. Loeliger and colleagues found no difference in mortality but a higher rate of reinfarction in untreated patients." They found the incidence of cardiovascular death in the treated group to be $4.8 \%$ against $7 \cdot 2 \%$ in the placebo group. True enough this difference is not statistically significant, but taken together with the statistical significant reduction of reinfarction rate the flat statement that Loeliger et al. found no difference in mortality is in my mind a slightly biased expression.

Further on you say: "It is agreed that long-term anticoagulants are of no benefit to women." It is correct that the difference in the mortality reported by the international group in women of 0.86 per 100 patient/months in the treated group against 0.93 in the control group could easily have been due to chance. We reported ${ }^{3}$ the results of a controlled clinical trial of longterm anticoagulant therapy in women after their first infarction and could show a significant reduction in mortality and reinfarction rate in the treated group. Admittedly this is not a large study, including 159 patients only, but still by far the largest study of this treatment in women so far reported. It would not seem unreasonable to have at least referred to this study which throws some doubts on the old idea that anticoagulant therapy after infarction is effective in men only.

It seems to me fair to conclude that the majority of statistically well-controlled trials show a significant reduction in mortality and/or reinfarction rate in the treated group, that some studies have shown a trend in favour of the treated group, but that none has shown that the treated group did worse than the control group. But to state as your leader writer does that there is "... absence of any evidence of benefit" seems to be quite far from the truth. If one wants to abandon the long-term use of anticoagulants after myocardial infarction I should certainly use other arguments than "the absence of any evidence of benefit" of this treatment.

Such a leading article gives food for many thoughts. One is that I would have liked the article to be signed.-I am, etc.,

Chr. F. BORCHGREvinK.

Institutt for Almenmedisin,

Oslo, Norway.

REFERENCES

International Anticoagulant Review Group, Lancet, 1970, 1, 203

Aspenstrøm, G., and Korsen-Bengsten, K., Acto Medica Scandinavica, 1966, 176, 563 Borchgrevink, C. F., et al., British Medical four-
nal, 1968, 3, 571.

\section{Azathioprine in Psoriasis}

SIR,-Cytotoxic drugs are widely used in the treatment of psoriasis which has failed to respond to conventional topical regimens. Most dermatologists have used methotrexate, but there have recently been several disquieting reports ${ }^{1-4}$ of renal and hepatic damage following methotrexate therapy. Because of an earlier favourable report of 6-mercaptopurine in psoriasis $^{5}$ we have carried out a preliminary study of the closely related drug azathioprine (Imuran) in this disease.

Ten patients were studied, all of whom has disabling psorasis which had proved refractory to prolonged topical treatment. Methotrexate had earlier been given to six of these, but had been withdrawn in four because of failure to respond. In the remaining two methotrexate had been stopped because of toxic effects. The total daily dosage of azathioprine was 2.5 $\mathrm{mg} . / \mathrm{kg}$. body weight and this was continued for six weeks. None of the patients studied was on any other systemic treatment for psoriasis during the period of study. The only local treatment prescribed was emulsifying ointment. All patients completed the six-week course of treatment.

Before beginning azathioprine haemoglobin, total and differential white cell counts and platelet counts, E.S.R., blood urea, and liver function tests were carried out, and were repeated at fortnightly intervals.

The results are summarized in the Table. Of the ten patients, five showed clearing of 